\title{
Hydrophobic Paper Architecture: Studies in the Sustainability of Impermanent Structures
}

\author{
SHELBY DOYLE \& LESLIE FOREHAND \\ lowa State University
}

"The problem with a tent is that when you use it you throw it away, so it's money that melts."

\section{-Alejandro Aravena}

The social project of architecture has long been fascinated with emergency and refugee housing as a primary unit of architectural and urban development. For decades, architects have proposed alternatives to the United Nations' blue tent cities that are the principal image associated with humanitarian aid and its resulting urbanism. During the $\mathbf{2 0 1 6}$ Venice Biennale Reporting from the Front, curator Alejandro Aravena challenges architects to reconsider the discipline's relationship to society's most urgent challenges. The ongoing European refugee crisis is one such 'Front' and this research examines the viability of an alternative to the polyvinylchloride (PVC) tarp as the default condition of emergency and refugee housing. The authors propose that waterproof paper surfaces and members, treated with a proprietary nano-coating can perform as well as traditional materials, but with reduced environmental impact and improved user comfort. A collaboration between researchers in Material Science and Architecture combines ongoing scientific research with digital design tools and methods. Following is a brief history of building with paper, an introduction to hydrophobic nano-coatings, and several fabricated prototypes. This project expands upon initial applications from the Tokyo Institute of Technology (2012) where researchers successfully prepared paper surfaces with a nano-particle coating, repelling water and maintaining structural integrity.
PROBLEM: TEMPORARY STRUCTURES WITH PERMANENT MATERIALS

Temporary structures are ubiquitous but highly wasteful in their material realization. This condition has far-reaching consequences from provisional refugee housing to disposable festival structures to trade fair booths. While these temporary structures are not designed for obsolescence due to their short-term needs, they are often highly resistant to decomposition. This unnecessary permanence manifest in their materiality, typically utilizing cheap, toxic, and non-biodegradable materials.

Tarpaulins or tarps are commonly distributed as lightweight shelter for refugees and disaster relief victims, and are chosen because of their low material cost and lightweight shipping. These plastic sheets are made of polyvinylchloride (PVC) a petroleum based compound that is non-biodegradable and leaches toxins into soils and water. While these plastics are incapable of bacterial decomposition, they are susceptible to mechanical failure due to other forces of nature specifically ultraviolet degradation from sun exposure breaks the molecular bonds of plastic, causing a large piece to breakdown into many little pieces, but never fully degrading. This mechanical failure often occurs before the end of its use. For example, most refugees remain in camps for nine to twenty-four months; these tarps typically fail within six months, requiring several more tarps and thus harmful PVC's to successfully shelter for the duration of the refugee's temporary accommodations. Hydrophobic paper presents an opportunity to challenge these shortcoming and to generate innovative materiality for temporary shelter. (Oxfam Report, 2008)

PRECEDENT: PAPER AS BUILDING MATERIAL

The material limitations of paper have not prevented architects and designers from integrating it into humanitarian designs. Pritzker recipient Shigeru Ban is noted for his use of paper as a building material, and began investigating the paper tube as a structural member in 1986. Ban leveraged the strength of the paper tube and its nearly universal availability to develop a series of projects: ranging from emergency shelter in India to refugee housing in Rwanda. Despite these successes, water remains a challenge for building with paper, and Ban's work employs multiple paper waterproofing techniques. One strategy is to coat the structures with polyurethane thereby 
augmenting the waterproofing film already applied to the tubes for their use in fabric storage. Another strategy uses tarps as the primary exterior envelope while the tubes serves as structural members. Locally sourced and sustainable structural framing help to keep costs low and prevent theft, however PVC poses challenges to occupant comfort and material disposal as the resulting coated paper tube can longer be recycled. (McQuaid, 2006)

While limiting the growing amount of construction waste through recycling is considered ideal, this is not always a viable option. With regards to refugee camps, inappropriate waste removal is a primary concern. In the absence of formal waste management strategies, inhabitants often resort to burning or burying their waste along the edges of the encampments unintentionally contaminating water supplies. When burned, the plastics present in tarps and other plastic coatings emit a gas that is hazardous to health. Eliminating the harmful repercussions of plastic disposal within these environments is a primary goal of this project. (Oxfam Report, 2008)

\section{PRECEDENT: EMERGENCY SHELTER + REFUGEE HOUSING}

Despite their shortcomings, PVC tarps are ubiquitous and inexpensive to ship. This research began with an extensive cataloging of previous design proposals for the development of emergency shelter and refugee housing. The aim was to extract some of the reasons why the design community, despite contributing extensive number of proposals, has yet to really engage in changing the delivery of emergency shelter and refugee housing. (Figure 1)

Through case study research several challenges were identified as external to design - such as those of the humanitarian aid complex: politics, funding, logistics, policy, security, and prioritization of sites. These systems are far more tangled and complicated than the scope of this project. Rather, this work focuses on the design and material properties of the shelters: weight, shipping, construction, and availability. As a result, the design intent of this research is to produce extremely lightweight (paper), easy to ship (flat or in rolls), easy to construct, readily available tents which are waterproofed through non-toxic nano-coatings making local disposal possible.
Homeless Emergency Shelter

Designer: Elkins DIY

Office/Location: Stanwood,WA

Year: 2012

Material Properties

Materials Used: 4 sheets $4 \mathrm{~mm}$ coroplast, 8 " plastic zip ties, and 2" colored duct tape Strength: Durable, flexable, and waterproof Cost Effective: $\$ 100$ Material Cost Eco Efficient: No

Breathable: Limited to small vent openings Transparent: Limited to door opening

UV Resistant: Yes

Scratch Resistant: Yes

Reflective: Yes

Dimensions: 40 " wide, 81 " long, 38 " tall (pre-cut assembly available in $4^{\prime} \times 8^{\prime} \times 1$ " package)

Assembly: Construction required, pre cut system could expedite this

Weight: 23 lbs

Occupancy: $1-2$ adults

Use Intent: Emergency Shelter

Delivery Method: Elkins DIY envisions mass shipping available with 50 shelters per pallet

Current Applications: Still in research and development stage.

https://groups.yahoo.com/neo/groups/TheConfederateCoalition/conversations/topics/7877
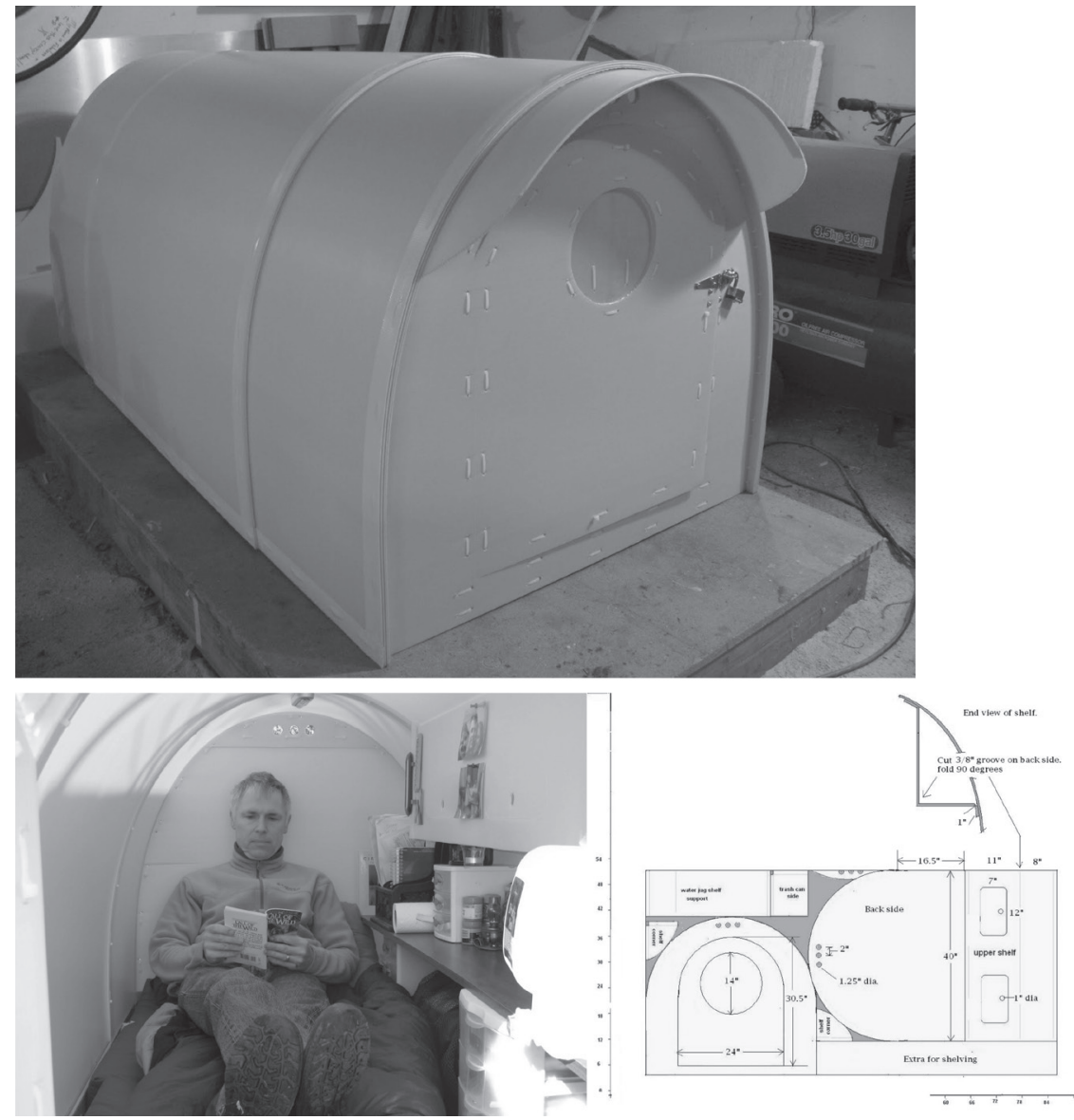

Figure 1: Above is an excerpt from the evalution of one hundred fifty proposals for emergency shelter and refugee housing. Each project was categorized by the same material properties, size, weight, cost, and whether it has actually been constructed. This allowed for a comparative study of reoccurring design challenges. 


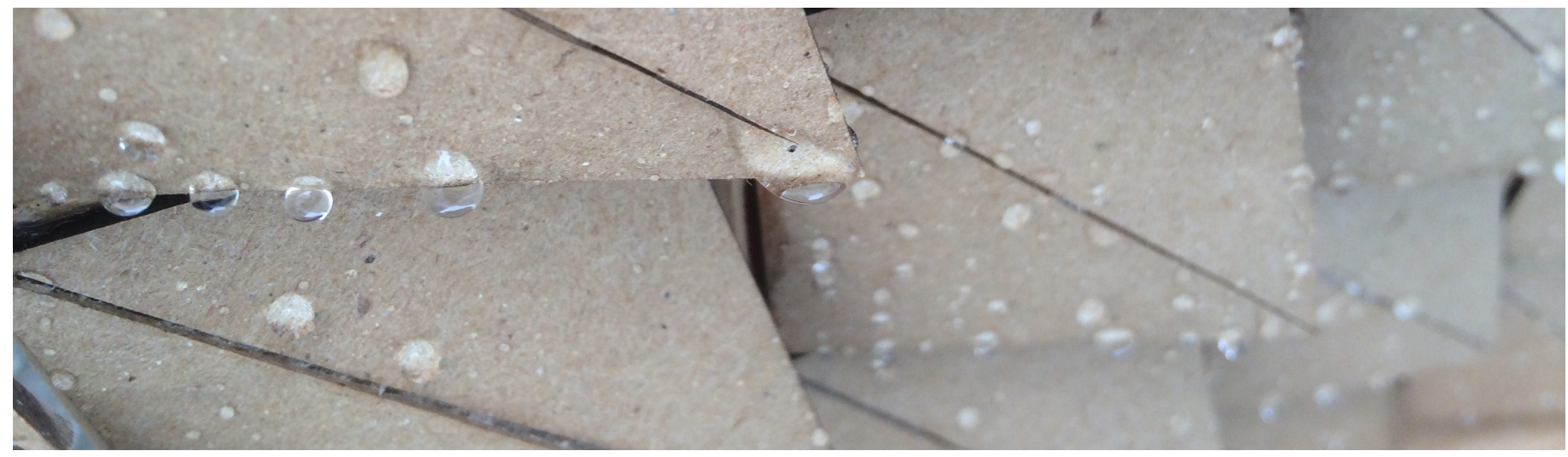

Figure 2: Above is a detail photo of scale chipboard model after a rainstorm that was treated with a proprietary non-toxic hydrophobic nano-coating developed at lowa State University. Use of this nano-coating at full scale was not possible due to current cost and availability.
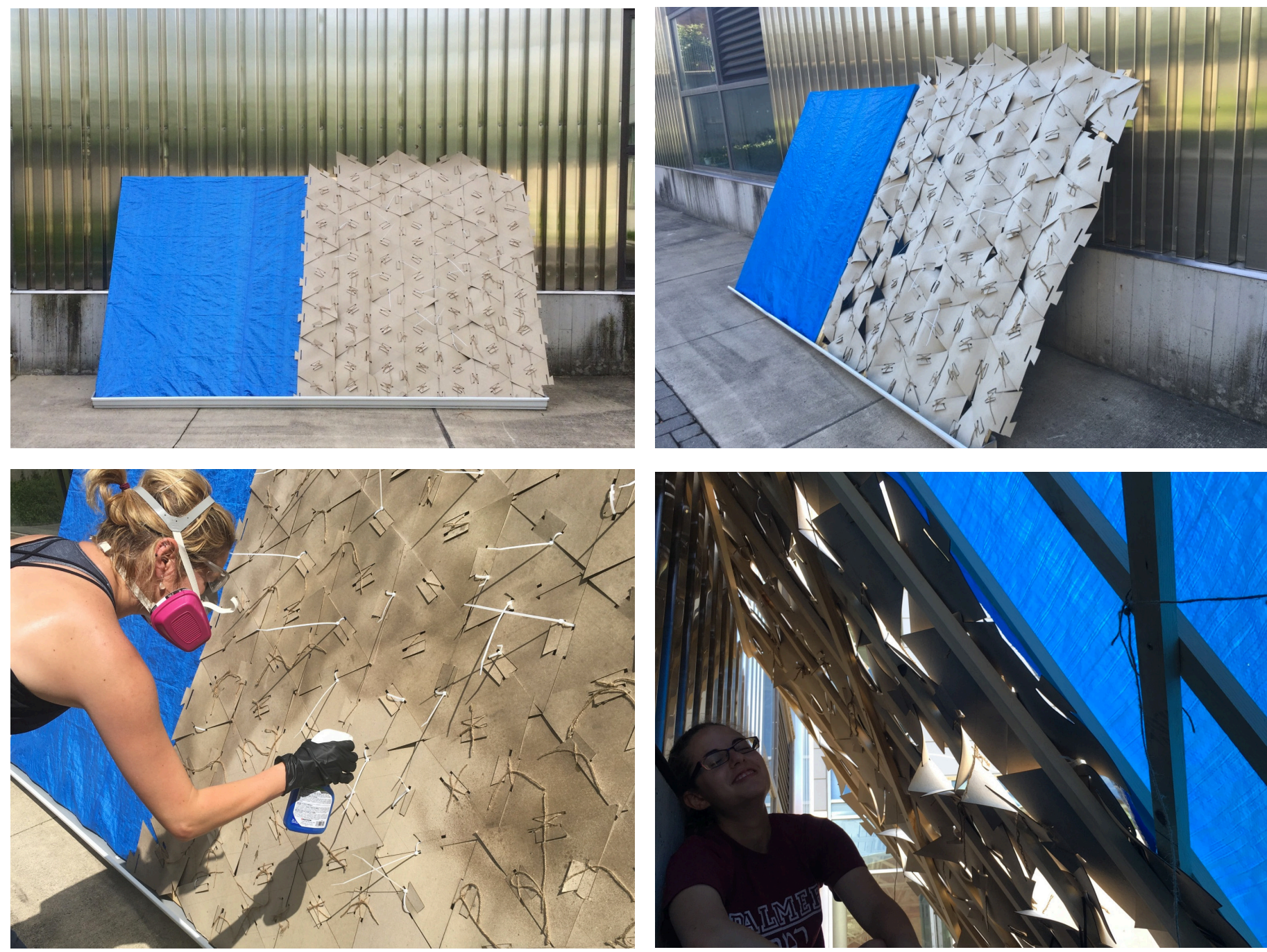

Figure 3: Paper wall process. Top right: Initial installation of tarp and paper walls. Bottom left: Hydrophobic treatment with commercially available nanocoating. Top left: After two rainstorms. Bottom right: Quality of light below the walls. 


\section{PROPOSED SOLUTION:}

\section{HYDROPHOBIC COATINGS FOR PAPER STRUCTURES}

This project applies hydrophobic coatings to paper skins and membranes, eliminating the need for plastic tarps and toxic coatings as solutions for waterproofing temporary structures. By applying a nano-thin coating that is 'rough' on the molecular level, water droplets cannot penetrate the material due to increased surface tension and thus are shed. (Figure 2) Unlike polyurethane coatings or paints, nano-coatings cause no noticeable property changes. After treatment, the paper has the same structural and performative qualities. Paper nano-coatings have great potential to produce a variety of desirable material effects for architects. By manipulating surface structures on the molecular level, water filtration surfaces and extreme protection against degradation can be fine-tuned. Working in conjunction with material scientists, multiple paper products, treatment methodologies, and design iterations are presented in this paper. Designs were evaluated with $\mathrm{HOBO}$ sensors for temperature and humidity reduction in relation to traditional PVC tarps. Nano-coatings could have wide reaching impacts across design scales. Specifically, the introduction of an alternative to the polyvinylchloride tarp as the default condition of emergency and refugee housing could present future design opportunities

Water repellent paper shows great potential in application across various fields, and this investigation applies it to the ecological challenges of temporary and semi-permanent structures. Paper is a similarly valued and weighted resource as a PVC tent, folding and stacking easily to meet the weight requirements for shipping and assembly. This research presents a composite of nanoparticle coatings and paper component modules that are assembled into full-scale temporary structures. This process allows for locally sourced sustainable materials (paper) and results in increased habitation duration through repair and renovation. This nano-particle treatment, which is sprayed on the paper in a controlled environment, does not contribute negatively to the environment: it can be burned after its use without producing harmful toxins. This system integrates hydrophobic paper surfaces and soy based biodegradable plastic hardware (under development), providing adequate temporary shelter that can be similarly deployed in the event of a disaster, and renovated as necessary.

Hydrophobic applications have design precedents. In 2001, Andrew Parker and Chris Lawrence, interdisciplinary scholars in the UK, published a paper in Nature titled 'Water Capture by a Desert Beetle'. This beetle, found in South Africa's Namib Desert, survives the harsh dune conditions by collecting fog into larger droplets on the insect's wings in alternating hydrophobic and hydrophilic regions. The beetle's strategy for hydration is simple: its bumpy back, when tilted with its back legs higher than its front, collects water and drips larger drops down to the beetle's mouth. Three formal elements: the angle of the beetles back, the size of the bumps, and the alternating hydrophobic and hydrophilic regions, permit this beetle to survive. This paper instigated commercial applications such as 'water-trapping tents and building coverings, or in water condensers and engines' and inspired countless biomimetic design proposals within the design disciplines. (Parker, 2001)
Hydrophobic nano-coatings employ similar concepts at a microscopic scale. By manipulating particles on the molecular level, bumps are 'grown' on the surface, creating a near invisible barrier of air over the treated surface and forcing water to slide over top the material below. This treatment repels water, causing the droplets to rebound upon impact, avoiding absorption. These coatings are currently composed of a range of materials, such as manganese oxide polystyrene, precipitate calcium carbonate, silica nano-coatings and beyond, and range in capabilities, toxicities and affordability. This technology is currently available to the public in varying forms, commonly used to guard valuable electronic devices from water damage and coat outdoor furniture fabrics to resist water saturation and subsequent mold problems. There are additional proposed architectural applications, such as protecting metal hardware from erosion and preventing residue build up on glazing. (Hu, 2001) (Lin, 2009)

\section{PROTOTYPE 1: PAPER WALL Intent}

The initial investigation sought to compare heat and humidity reduction between a typical PVC tarp and a surface constructed of paper (chipboard) and hydrophobic nano-coatings.

Design + Process (Figure 3)

Early attempts were made to design and construct a self-structured paper module. After several failures this was abandoned in favor of a simple lumber frame to which the PVC and paper were affixed using either zip ties or staples. Individual units were designed as a tab and slot system which allowed individual modules to aggregate into any size of surface area. Early attempts to use the laser cutter were identified as an issue by the material science collaborators as the laser burns the edges of the paper change its material properties and alter the application of the nano-coatings. Therefore future investigations will investigate modules that are produced with blades via hand-cut or CNC drag knife.

\section{Analysis}

A series of Onset's HOBO MX1101 data loggers measured and transmitted temperature and relative humidity data wirelessly to mobile devices via Bluetooth Low Energy (BLE) technology. Graphs were created from the collected data and alternative designs generated based on these analyses.

\section{Results}

HOBO data indicated a negligible difference in heat and humidity reduction between the PVC and paper wall. An informal survey of users noted that the quality of light and general comfort was higher beneath the paper wall.

\section{PROTOTYPE 2: PAPER TENT Intent}

The second investigation sought to resolve the challenges identified in the wall project and produce a two-side freestanding tent structure relying only upon copy paper and staples. 

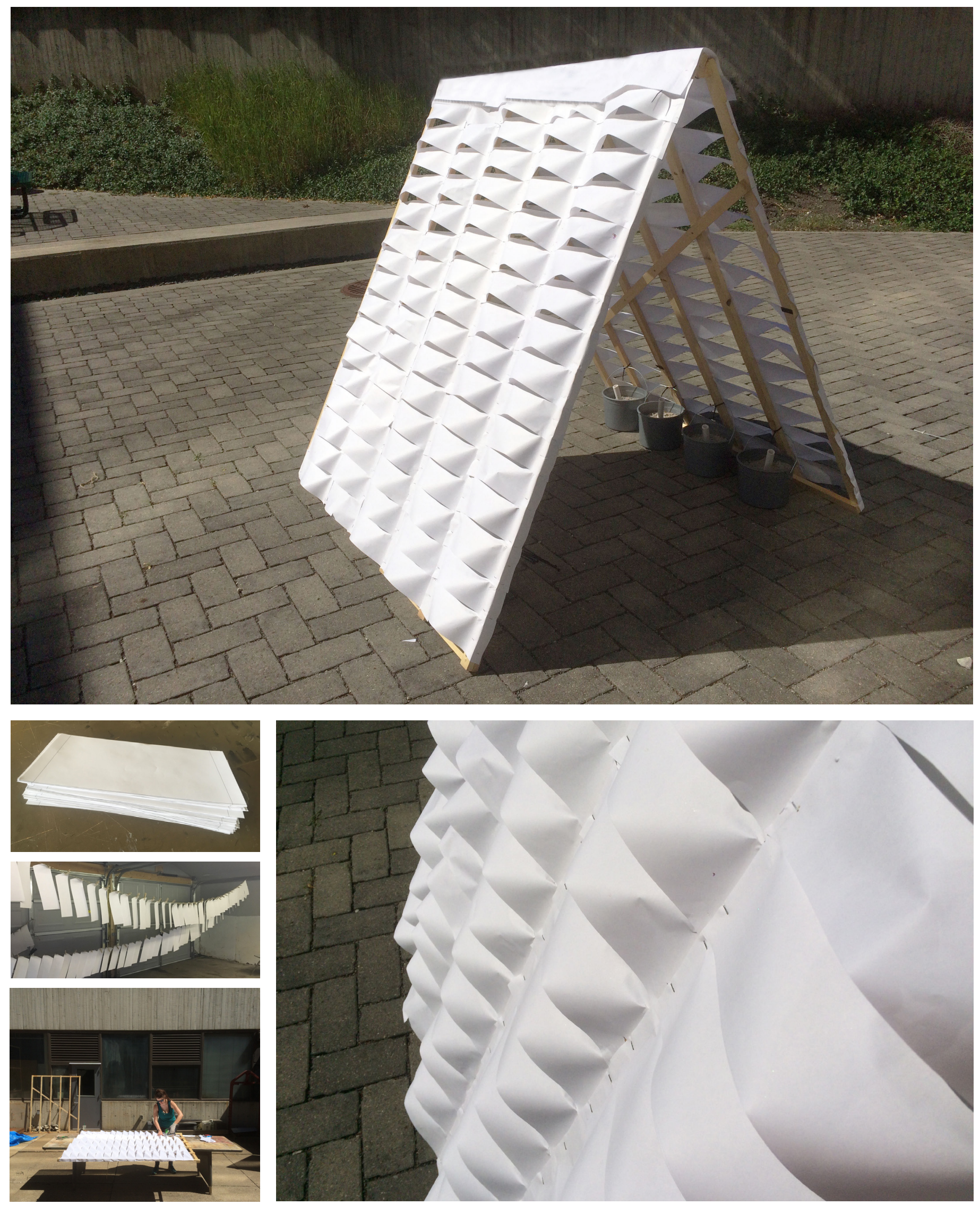

Figure 4: Paper tent process. Top: Initial tent installation. Bottom left: Hydrophobic treatment with commercially available nano-coating. Bottom right: Detail of venting and hardware. 
Design + Process (Figure 4)

Utilizing existing frames from the initial paper wall studies, a paper tent was constructed in two hours. Acknowledging the failures that occurred with the geometry and materiality of the chipboard wall, the tent employed a simple shingle technique from hydrophobiccoated letter-sized copy paper. Each shingle was printed, cut with a paper cutter, and pre-treated individually, rather than assembled and then treated, eliminating opportunities for water absorption. The shingle bubbled outward to encourage airflow.

Results

The copy paper failed mechanically (ripped) before the waterproofing failed. The tent survived two intense thunderstorms. A third with high winds ripped the paper at the staple connection. Future designs will reconsider the thickness of the paper used as well as the mechanical fasteners.

\section{PROSPECTIVE: DIFFERENT NANO COATINGS}

The introduction of non-toxic nano-coatings into architecture from the scale of industrial design to construction has wide reaching ramifications. Coated paper is already widely used and distributed in products as varied as cereal boxes, magazine pages, and food containers. In the building trades, paper is embedded with plastics and used in building wraps such as Tyvek, serves as a backer to batt insulation, and as a component in a variety of adhesive products. By retooling coated paper products to be biodegradable, burnable, or even recyclable, nano-coatings could have wide reaching impacts across design scales.

\section{CONCLUSIONS}

This research project demonstrated how nano-coatings can provide alternatives to the polyvinylchloride tarp as the default condition of emergency and refugee housing. Broadly speaking, nano-coatings have the potential to transform architectural materials and introduce new opportunities for innovative designs. By manipulating surface structures on the molecular level, properties such as water filtration and extreme protection against degradation can be finetuned. While this project utilized affordable (yet toxic) options commercially available to explore the viability of material treatments, future investigations hope to fully engage the propietary non-toxic coatings under development at lowa State University.

\section{ACKNOWLEDGMENTS}

This research was made possible by funding from the lowa State University Center for Building Energy Research, the Daniel J. Huberty Faculty Fellowship, the support of the lowa State University Department of Architecture and College of Design and the work of Master of Architecture Candidates and Graduate Research Assistants Andrew Meyer and Zhenhua Yin, as well as the work of Bachelor of Architecture Candidates Makayla Natrop and Sarah Schneider. Non-toxic nanoparticle coatings used in this project are the proprietary work of Martin M. Thuo, Assistant Professor of Material Science, lowa State University and PhD Candidate Stephanie Oyola-Reynoso. Thuo is partially supported by a Black and Veatch Fellowship. Oyola-Reynoso is supported in part by a GMAP fellowship from lowa State University and startup funding to Thuo.

\section{ENDNOTES}

1. Domestic and Refugee Camp Waste Management Collection and Disposal, 2008 . http://policy-practice.oxfam.org.uk/publications/domestic-and-refugee-campwaste-management-collection-and-disposal-126686domestic-and-refugeecamp-waste-management-collection-and-disposal-126686

2. Hu, Z.; Zen, X.; Gong, J.; Deng, Y. (2009). "Water resistance improvement of paper by superhydrophobic modification with microsized $\mathrm{CaCO} 3$ and fatty acid coating". Colloids and Surfaces A: Physicochemical and Engineering Aspects 351: 65. doi:10.1016/j.colsurfa.2009.09.036.

3. Lin, J.; Chen, H.; Fei, T.; Zhang, J. (2013). "Highly transparent superhydrophobic organic-inorganic nanocoating from the aggregation of silica nanoparticles". Colloids and Surfaces A: Physicochemical and Engineering Aspects 421: 51. doi:10.1016/j.colsurfa.2012.12.049.

4. McQuaid, Matilda. Shigeru Ban. Phaidon Press, 2006.

5. Ogihara, Hitoshi, Jing Xie, Jun Okagaki, and Tetsuo Saji. 2012. "Simple Method for Preparing Superhydrophobic Paper: Spray-Deposited Hydrophobic Silica Nanoparticle Coatings Exhibit High Water-Repellency and Transparency." Langmuir : the ACS journal of surfaces and colloids 28(10), pp.4605-4608.

6. Parker, Andrew R and Lawrence, Chris R. 2001. "Water Capture by a Desert Beetle." Nature 414, 33-34 